\title{
Prediction of leakage in the fixed mechanical seal
}

\author{
Anatoly A.Asheichik ${ }^{1, *}$, and Mohammad Reza Bahrami $^{1}$ \\ ${ }^{1}$ Peter the Great St.-Petersburg polytechnic university, St.-Petersburg, Russian Federation
}

\begin{abstract}
The questions of influence of the shape of contact surfaces on leakages through rubber seals in fixed connection of subassemblies are considered in the article. It is known from practice of operation of seals of various designs that the shape of contact surfaces and consequently also the shape of diagram of stresses in a contact zone considerably influences on value of leaks Linking leakage magnitude and distribution of contact stresses enables, firstly, more precisely calculate the amount of leakage for existing seals, and, secondly, to optimize the shape of the seals in their design in each case. As the result of experimental studies on the introduction of the rubber gasket ring fixed indenters different profiles found that by optimizing the shape of the indenter magnitude of leakage can be reduced by 10 times.
\end{abstract}

\section{Introduction}

Leakage through a circular fixed mechanical seal can be defined by the formula [1]

$$
Q=\frac{\pi}{6 \ln R_{1} / R_{2}} \frac{\Delta p}{\eta} \psi_{0} R_{z}^{3} \exp \left(-\frac{3 \sigma_{n}}{k E}\right)
$$

where $Q$ is leakage through the seal $R_{1}$ and $R_{2}$ - the outer and inner radii of gaskets; $\Delta p$ is pressure drop; $\eta$ is the viscosity of the sealing medium $\psi_{0}$ is the coefficient of micro-roughnesses form; $R_{Z}$ is the sealing surface roughness parameter; $\sigma_{n}$ is the normal stress on the contact point; $E$ is the seal modulus; $k$ is the coefficient taking into account the specific features of the physical model 0.05 to 0.3 .

Formula (1) derived from the Navier-Stokes equations for laminar flow of gas without considering the centrifugal forces. From the analysis of the above expression for leak detection, follows that they are largely dependent on the magnitude of the stresses in the zone of contact of the seal with the sealing surface. The magnitude of this stress is known [1-4], which depends on the preload pressure $p_{k o}$ and in some designs of seals depends on the drop pressure of sealed medium $\Delta p$. Usually in the formula (1) the average value of the normal stress is using [5-7].

From experimental practices of various designs of seals [8-10] is known that the shape of the contact surfaces and, consequently, the shape and stress distribution in the contact area significantly affects the amount of leakage. In literature, however, practically there is no information about the effect of contact stress distribution along the width of the contact area on leakage. Thus establishing a relation between leak size and distribution of contact stresses will allow firstly to better calculate the amount of leakage for existing seals and secondly to optimize the shape of the seals in their design in each case.

\section{Apparatus for investigations and test method}

Scheme of apparatus for studying leakage through mechanical seal is shown in Fig. 1. The appearance of the elements of the structure with photographs of the ring indenter and their schemes are shown in Fig. 2.

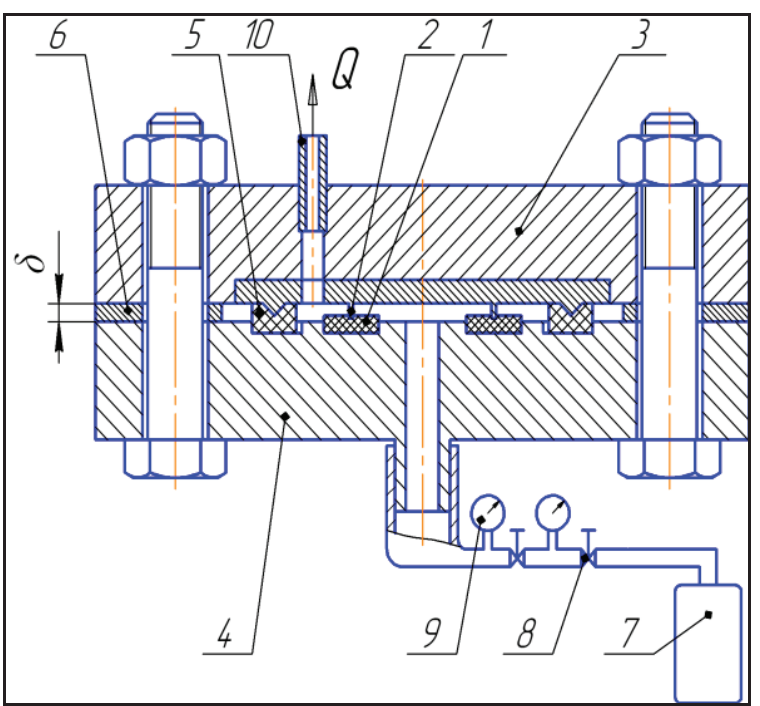

Fig. 1. The apparatus for leakage studying through mechanical seal.

The object of the study is a fixed mechanical ring seal 1 . In tests, the rubber O-ring clamped between two steel flanges 3 and 4 . In the inner cavity seal supplied pressurized air, $0-1.5 M P a$. Air was supplied from a gas cylinder 7, where its pressure was $15 \mathrm{MPa}$ through the gas reducer 8. Accuracy of adjustment and

\footnotetext{
* Corresponding author: aseichik52@,mail.ru
} 
measurement of overpressure was $0.01 \mathrm{MPa}$. leakage measured from the outside of the gasket (10 fitting).

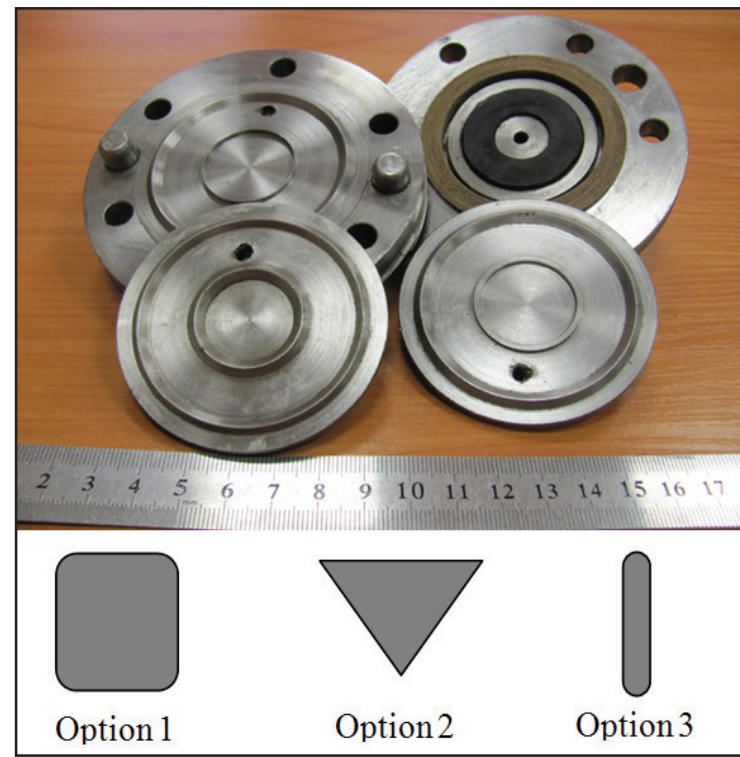

Fig. 2. Appearance of the elements of the structure and the scheme of indenters variants.

The dimension of the rubber O-ring with the rectangular section 1 is as follows: outer diameter of 36 $m m$, inner diameter of $16 \mathrm{~mm}$ and height of $4 \mathrm{~mm}$. It was placed in an annular groove on the steel flange 3 with the depth of $2.6 \mathrm{~mm}$ and the diameter is equal to the diameter of the gasket and glued. In the opposite flange laid removable ring indenters 2 of different shapes (3 options, see Fig. 2).

In the first embodiment, it was a rectangular protrusion $1.8 \mathrm{~mm}$ wide with radii of $0.3 \mathrm{~mm}$ at the edges (width of the flat portion $1.2 \mathrm{~mm}$ ). In the second embodiment, the protrusion section is an equilateral triangle with angles at the apex of $90^{\circ}$ and the radius at the tip of $0.5 \mathrm{~mm}$. In the third embodiment, it was rectangular protrusion $0.4 \mathrm{~mm}$ wide with the radius of $0.2 \mathrm{~mm}$ at the top. The roughness surface of protrusion corresponded to $R_{a}=1.25 \mu \mathrm{m}$. Static modulus of rubber was $E=3.2 \mathrm{MPa}$. Average diameter of the protrusions in all cases was $25.8 \mathrm{~mm}$, and the height was $1.8 \mathrm{~mm}$. The preload pressure $p_{k o}$ was created by six bolting joints which are spaced evenly over flanges. Movement was regulated and measured by instrumental measurement tiles 6 (see Fig. 1) with the accuracy of $0.01 \mathrm{~mm}$.

During the test, the rubber O-ring was placed in the groove on the steel flange. By means of bolting and measuring plates 6 was set in the rubber specified minimum implementation $\delta$ of the annular protrusion indenter, made from the first embodiment. Then, through the gas reducer in the internal cavity of the seal was applied the specified minimum air pressure and the leakage was measured. After this, the air pressure was increased and leakage measurement was repeated. After measuring the leakage over the entire range of pressures, the implementation value $\delta$ has been increased to the following amount, and all measurements were repeated.

\section{Results of experimental studies}

As the result of the experimental studies were obtained the dependence of leakage from excessive pressure at different values of the implementation of the protrusion in the rubber. These dependencies for the first variant form of the indenter are presented in Figures 3-5.

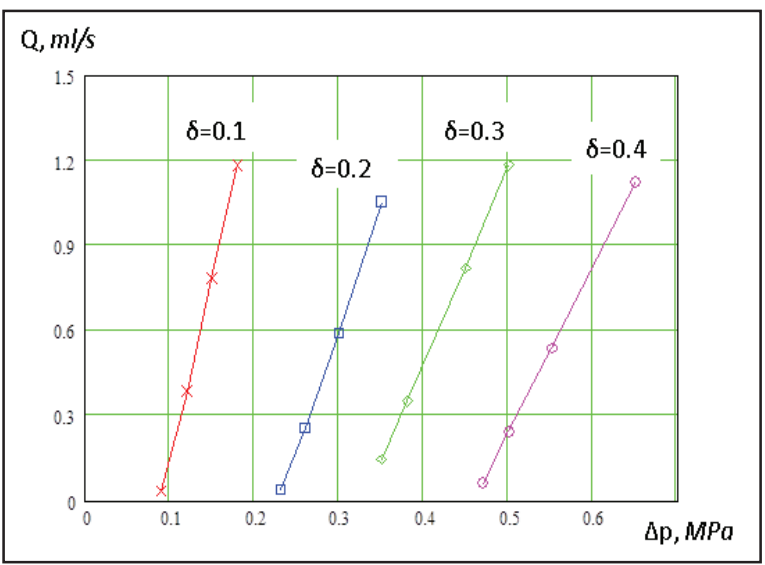

Fig. 3. Dependence of leaks from the pressure drop at different implementations $\delta$ in the rubber indenter made of embodiment 1.

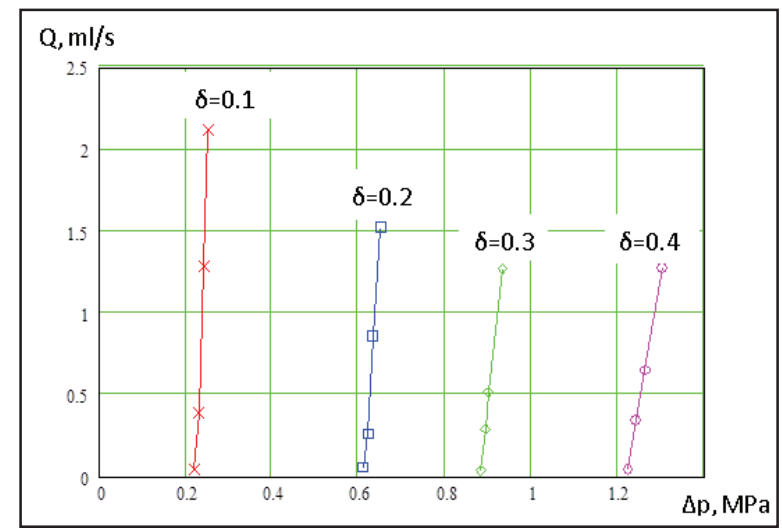

Fig. 4. Dependence of leaks on the pressure drop for various insertions of $\delta$ into the rubber.

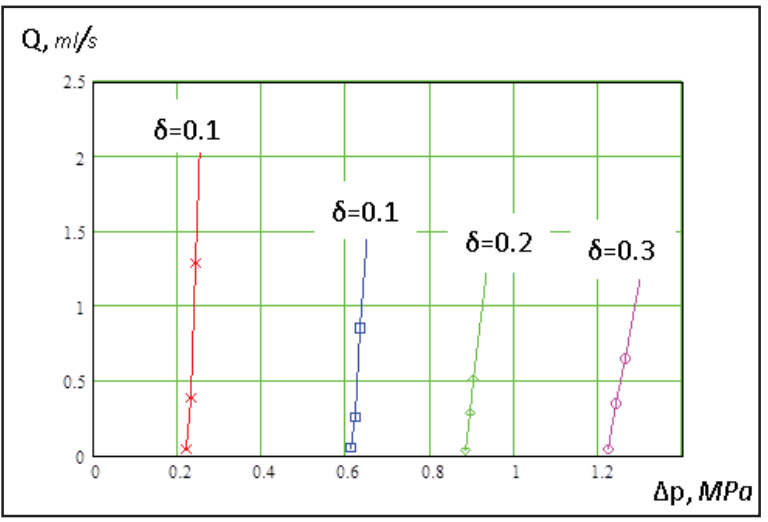

Fig.5. Dependence of leakages on the pressure drop for various insertions of $\delta$ into the indenter rubber manufactured according to the variant 3 . 
Table 1. Results of geometric similarity coefficient calculation.

\begin{tabular}{|c|c|c|c|c|c|}
\hline$\delta, m m$ & $R_{l}, m m$ & $R_{2}, m m$ & $R_{1} / R_{2}$ & $\ln R_{1} / R_{2}$ & $\left(\ln \frac{R_{1}}{R_{2}}\right)_{1} /\left(\ln \frac{R_{1}}{R_{2}}\right)_{j}$ \\
\hline \multicolumn{6}{|c|}{ 1st embodiment } \\
\hline 0.1 & 13.72 & 12.08 & 1.1358 & 0.12646 & 1 \\
\hline 0.2 & 13.77 & 12.03 & 1.1446 & 0.13506 & 1 \\
\hline 0.3 & 13.80 & 12.00 & 1.1500 & 0.13977 & 1 \\
\hline 0.4 & 13.80 & 12.00 & 1.1500 & 0.13977 & 1 \\
\hline \multicolumn{6}{|c|}{ 2nd embodiment } \\
\hline 0.1 & 13.20 & 12.60 & 1.0476 & 0.04650 & 2.72 \\
\hline 0.2 & 13.30 & 12.50 & 1.0640 & 0.06204 & 2.17 \\
\hline 0.3 & 13.50 & 12.30 & 1.0976 & 0.09313 & 1.50 \\
\hline 0.4 & 13.60 & 12.20 & 1.1148 & 0.10868 & 1.29 \\
\hline \multicolumn{6}{|c|}{ 3rd embodiment } \\
\hline 0.1 & 13.06 & 12.74 & 1.0251 & 0.02479 & 5.10 \\
\hline 0.2 & 13.10 & 12.70 & 1.0315 & 0.03101 & 4.37 \\
\hline 0.3 & 13.10 & 12.70 & 1.0315 & 0.03101 & 4.50 \\
\hline 0.4 & 13.10 & 12.70 & 1.0315 & 0.03101 & 4.50 \\
\hline
\end{tabular}

Analysis of the results showed that for all variants forms of indenters and for all values of the implementations of the indenter in the rubber observed linear dependence of the leakage of the sealed medium pressure drop that corresponds to the formula (1).

However, the obtained dependencies does not allow to compare the results of experiments with various embodiments indenters since radiuses $R_{1}$ and $R_{2}$ (macrogeometry), used in formula (1), in these three structures differ sharply. Values of these radii and the ratio $\left(\ln R_{1} / R_{2}\right)_{1} /\left(\ln R_{1} / R_{2}\right)_{j}$ which may be called the geometric similarity ratio, in comparable embodiments with different implementations of the indenter in the rubber $\delta$ are given in table 1 . The index $j$, here corresponds to the embodiment which is compared with the first embodiment, that is selected as the basic embodiment.

The use of geometric similarity coefficient allows lead dependencies obtained experimentally for different indenters to the basic embodiment (embodiment 1). This alignment makes it possible to eliminate the influence of all factors on the leakage except concentration of the stress created by the indenter in embodiments 2 and 3 . From the leakage calculations regarding the similarity factor we can say leak in the second embodiment is approximately 2 times less than in the first (basic) embodiment and in the third embodiment, the difference reaches 10-times.

Analysis of the expression (1) allows determining the excess of the maximum stress $\sigma_{\max }$ over medium stresses $\sigma_{m}$. This can be accomplished by the use of the results of the two series of experiments. In the first series for the base embodiment (first), $\sigma_{\max }=\sigma_{m}$. In the second series, $\sigma_{\max }$ should significantly exceed the average stress, achieved for the second and third embodiments form indenters. Transforming the formula (1), we obtain

$$
\sigma_{\max }-\sigma_{m}=\frac{1}{3} \cdot \mathrm{kE} \cdot \ln \left\{\frac{\mathrm{Q}_{1}}{\mathrm{Q}_{\mathrm{j}}} \cdot \frac{\Delta p_{j}}{\Delta p_{1}} \cdot \frac{\left(\ln \frac{R_{1}}{R_{2}}\right)_{1}}{\left(\ln \frac{R_{1}}{R_{2}}\right)_{j}}\right\},
$$

where the value

$$
B=\frac{Q_{1}}{Q_{j}} \cdot \frac{\Delta p_{j}}{\Delta p_{1}} \cdot\left(\ln \frac{R_{1}}{R_{2}}\right)_{1} /\left(\ln \frac{R_{1}}{R_{2}}\right)_{j},
$$

can be called the general similarity coefficient.

Value $\sigma_{m}$ can be defined as

$$
\sigma_{m}=E / \varepsilon,
$$

where $\varepsilon$ is the relative deformation which in this experiment can be defined as

$$
\varepsilon=\delta / h \text {, }
$$

where $h$ is the thickness of the rubber gasket $h=4 \mathrm{~mm}$.

Then, using the experimental values of the pressure drops and the related leak from Fig. 2 and of similar 
Table 2. The calculation results of the experimental ratio $\sigma_{\max } / \sigma_{m}$

\begin{tabular}{|c|c|c|c|c|c|c|c|c|c|}
\hline $\begin{array}{c}\delta \\
m m\end{array}$ & $\begin{array}{c}Q_{1} \\
\mathrm{ml} / \mathrm{s}\end{array}$ & $\underset{\mathrm{ml} / \mathrm{s}}{Q_{j}}$ & $\begin{array}{c}\triangle p_{1} \\
M P a\end{array}$ & $\begin{array}{c}\Delta p_{j} \\
M P a\end{array}$ & $B$ & $\varepsilon$ & $\begin{array}{c}\sigma_{m} \\
M P a\end{array}$ & $\begin{array}{c}\sigma_{\max } \\
M P a\end{array}$ & $\sigma_{\max } / \sigma_{m}$ \\
\hline \multicolumn{10}{|c|}{ comparing the second embodiment with the base (first) } \\
\hline 0.1 & 1.280 & 0.640 & 0.24 & 0.18 & 4.08 & 0.025 & 0.08 & 0.155 & 1.93 \\
\hline 0.2 & 1.260 & 0.540 & 0.93 & 0.44 & 2.40 & 0.050 & 0.16 & 0.207 & 1.29 \\
\hline 0.3 & 1.270 & 0.724 & 1.30 & 0.88 & 1.78 & 0.075 & 0.24 & 0.271 & 1.13 \\
\hline \multicolumn{10}{|c|}{ comparing the third embodiment with the base (first) } \\
\hline 0.1 & 1.280 & 0.780 & 0.24 & 0.15 & 5.10 & 0.025 & 0.08 & 0.168 & 2.10 \\
\hline 0.2 & 1.260 & 0.588 & 0.93 & 0.30 & 4.37 & 0.050 & 0.16 & 0.218 & 1.36 \\
\hline 0.3 & 1.270 & 0.820 & 1.30 & 0.45 & 4.50 & 0.075 & 0.24 & 0.287 & 1.20 \\
\hline
\end{tabular}

dependencies for indenters for embodiments 2 and 3, and taking $k=0.05$, we obtain experimental ratio $\sigma_{\max } / \sigma_{m}$. All results of these calculations are summarized in Table 2.

\section{Conclusions}

As the result of experimental studies on the implementation of circular indenters of various profiles in the rubber gasket fixed found that by optimizing the shape of the indenter, magnitude of leakage can be reduced by 10 times. Establishing relation between the leak and the size of the contact stress distribution allows more precisely calculate the amount of leakage for existing seals, as well as to optimize the shape of the seals in their design.

\section{References}

1. A.I. Golubev, L.A. Kondakov, seals and sealing machines: Directory (Mechanical engineering, Moscow, Russia, 1986)

2. S.O. Lazarev, A.A. Asheichik, V.L.Polonsky, Investigation of the influence of stress distribution in the contact area on the leakage through the rubber seal in a fixed joint (Publishing House of STU, St. Petersburg, Russia, 311-312, 2006)

3. A.A. Asheichik, S.G. Chulkin, Experimental Mechanics (Polytechnical University Press, St. Petersburg, Russia, 2008)

4. A.A. Asheichik, V.L. Polonsky, Experimental study of elastomers and polymers for the oil industry, (Polytechnical University Press, St. Petersburg, Russia, 2015)

5. D.A. Denischenko, Determination of the activation energy of elastomers by the experimental-theoretical method (Week of Science SPbPU: Part 1, St. Petersburg, Russia 65-67, 2014)

6. A.A. Kukina, Prediction of the change in the strength properties of rubber in its thermal aging (Week of Science of SPbPU: Part 1, St. Petersburg, Russia 63-65, 2014)

7. S.O. Lazarev, V.L. Polonsky, Computational mechanics, (Publishing house of Polytechnic. University, St. Petersburg, Russia, 2007)

8. G.R. Khachatryan, B.V.Ratner, Investigation of the antifriction properties of metal-filled rubbers, Caoutchouc and Rubber, №6, 33-35 (1981)

9. G.M. Bartenev, Structure and relaxation properties of elastomers, Chemistry, Moscow (1979)

10. N.P. Kirillov, N.P. Burenin, V.I. Polyansky and others, Structures of mechanical seals for rotating shafts of centrifugal pumps of rocket refueling systems, Herald of MADI, №1, 18-22 (2012) 